http://dx.doi.org/10.18778/2196-8403.2012.12

\title{
EWA TURKOWSKA
}

\section{Literarische Bildung in der Krise}

Artykuł przedstawia problemy kształcenia literackiego germanistów, wynikające z kryzysowej sytuacji szkolnictwa wyższego, oraz poszukiwania możliwych rozwiązań. $\mathrm{Na}$ początku przedstawiono cele kształcenia literackiego i przyczyny problemów dydaktycznych. W głównej części artykułu zaproponowano możliwe środki zaradcze. Na przykładzie zastosowania hiperfikcji i blogów na lekcji przedstawiono na koniec, jak można dziś w sposób nowoczesny nauczać literatury.

Der Artikel setzt sich mit den Problemen der literarischen Bildung im Germanistikstudium auseinander, die sich aus der krisenhaften Situation an den Hochschulen ergeben, und mit möglichen Lösungswegen. Zu Beginn werden die Ziele der literarischen Bildung und die Ursachen der didaktischen Probleme besprochen. Im Hauptteil des Artikels werden Maßnahmen vorgeschlagen, die den erwähnten Schwierigkeiten entgegenwirken können. Am Beispiel der Arbeit mit Hyperfiktionen und Blogs wird am Ende aufgezeigt, wie ein moderner Literaturunterricht heutzutage gestaltet werden kann.

The article presents problems of literary education in German studies, arising from the crisis situation of higher education, and the search for potential solutions. At first, it discusses the aims of literary education and the causes of educational problems. The main part of the article suggests possible remedies. Finally, it is presented how to use hyperfictions and blogs in class in order to teach literature in a modern way.

Die Beschäftigung mit Literatur bildet eine der Grundlagen des neuphilologischen Studiums. Die Krise der Germanistik wirkt sich auf die Situation des deutschsprachigen Literaturunterrichts besonders gravierend aus. 


\section{Zu den Begriffen ,literarische Kompetenz ${ }^{6}$, ,literarisches Ler- nen', ,literarische Bildung'}

Das Ziel der Beschäftigung mit Literatur in und außerhalb der Schule liegt im Erwerb der literarischen Kompetenz. Sie bildet einen Teil der allgemeinen ästhetischen Kompetenz, die dem Menschen ein wichtiges Werkzeug ,zur Auseinandersetzung mit sich selbst (Individuation), mit den Mitmenschen (Sozialisation) und der Umwelt (Enkulturation)“ bietet (ABRAHAM / KEPSER 2006:50). ${ }^{1}$ Die literarische Kompetenz besteht aus vielen Teilkompetenzen, die von Literaturdidaktikern unterschiedlich klassifiziert und benannt werden. ${ }^{2}$ In der neueren Forschung hat sich die Einteilung in die literarische Rezeptionskompetenz, die Produktionskompetenz und die Handlungskompetenz durchgesetzt. Die erste meint die Fähigkeit, Literatur zu erfahren, zu verstehen und zu interpretieren. Mit der zweiten ist die Fähigkeit gemeint, mit literarischen Texten kreativ-gestaltend umzugehen: sie vorzutragen, dar-

1 An der Herausbildung der ästhetischen Kompetenz wirken neben der Literatur auch die bildende Kunst und die Musik mit. Die Herausbildung der literarästhetischen Kompetenz im Literaturunterricht ist allerdings vorwiegend rezeptionsorientiert. Während im Musik- und Kunstunterricht die eigene ästhetische Gestaltung der Lerner im Mittelpunkt steht (singen, musizieren, Bilder malen), bezog sich der Literaturunterricht in seiner Geschichte stets auf die Auseinandersetzung mit dem fertigen literarischen Text, die eigene literarische Produktion der Schüler spielte kaum eine Rolle. Die Aufwertung des kreativen Schreibens brachte erst der handlungsorientierte Ansatz der Literaturdidaktik in den 1980er Jahren (vgl. ABRAHAM / KePSER 2006:51-59).

2 Z.B. erwähnt HAAS (1997:35-37) sieben Teilkompetenzen: die Fähigkeit, in Kontakt mit Literatur zu treten, die emotive, kreative, emanzipatorische, ästhetische, kritische Kompetenz und die Projektionskompetenz. SPINNER (2006) nennt elf Aspekte literarischen Lernens, die als literarische Teilkompetenzen aufgefasst werden können, darunter u.a. die Fähigkeit, beim Lesen Vorstellungen zu entwickeln, die Fähigkeit zu subjektiver Involviertheit, zur genauen Wahrnehmung der sprachlichen Gestaltung, zum Nachvollziehen der Perspektiven literarischer Figuren, zum Umgang mit der literarischen Fiktion, zur Entwicklung des literarhistorischen Bewusstseins, zur Orientierung innerhalb der Vielfalt der literarischen Genres. Andere Begriffsbestimmungen finden sich bei J. Kreft, Ch. Garbe, E. Schön, G. Waldmann, vgl. ihre kurze Darstellung bei KuMSCHLIES (2008:18-41) oder JAZBEC (2009:63-65). Die literarische Kompetenz wird in Praxis Deutsch 33 (2006) von mehreren Autoren ausführlich besprochen. Für den fremdsprachlichen Literaturunterricht schlägt z. B. BURwITZ-MELZER (2007) ein Lesekompetenzmodell des literarischen Lesens vor, das zur Entwicklung der literarischen Kompetenz beiträgt. 
zustellen, eigene lyrische, epische bzw. dramatische Texte zu schreiben und vorzuspielen. Die Handlungskompetenz befähigt die Lerner zur Teilhabe am literarischen Leben, wobei die Fähigkeit zur literarischen Wertung und zur Kommunikation über Literatur als Teilbereich der Kultur eine wichtige Rolle spielt (vgl. ABRAHAM / KEPSER 2006:55-63). Diese Kompetenzen sollen die Leser dazu befähigen, mit literarischen Texten autonom umzugehen. Die literarische Kompetenz wird oft in Beziehung zur Lesekompetenz gesetzt, da das Lesen die Grundvoraussetzung für das Erreichen der Literaturkompetenz ist. Im Vergleich zur Lesekompetenz ist die literarische Kompetenz einerseits umfassender, weil sie nicht nur auf die Rezeption geschriebener Texte, sondern auch auf die Rezeption anderer medialer Formen der Literatur ausgerichtet ist und sich noch vor dem Lesenlernen zu entwickeln beginnt. Andererseits ist die literarische Kompetenz klar umrissen, denn sie ist nur auf den Bereich literarischer Texte bezogen und klammert Sachtexte aus (vgl. JAZBEC 2009:62). Die Leseförderung ist ein wichtiger Bestandteil des Literaturunterrichts. ${ }^{3}$ Aufgrund unterschiedlicher medialer Gestaltungsmöglichkeiten der Literatur (neben dem traditionellen Printmedium Buch auch als Hörbuch, als Film und in digitaler Form) trägt die Beschäftigung mit ihr auch zum Erwerb der Medienkompetenz bei (vgl. 6. Abschnitt). Der Weg, die literarische Kompetenz zu entwickeln, führt über das literarische Lernen. Die literarische Kompetenz wird fortschreitend in einem lebenslangen Prozess erworben, dank dem Umgang mit Literatur in allen medialen Formen in und außerhalb der Schule. Der Begriff ,literarisches Lernen“ ist subjektbezogen, offen und weit gefasst. Er meint neben der literarästhetischen Bildung auch den Beitrag der Literatur zur Persönlichkeitsbildung der Lerner. P. Büker bezeichnet das literarische Lernen als „schulische Lehr- und Lernprozesse zum Erwerb von Einstellungen, Fähigkeiten, Kenntnissen und Fertigkeiten, die nötig sind, um literarisch-ästhetische Texte [...] zu erschließen, zu genießen und mit Hilfe eines produktiven und kommunikativen Auseinandersetzungsprozesses zu verstehen“ (zit. nach ABRAHAM / KEPSER 2006:82). Einen beträchtlichen Teil des literarischen Lernens nimmt die literarische Bildung ein. Der Begriff ist objektbezogen und auf die materielle Seite des Lernprozesses in der Schule gerichtet (Lerninhalte). Die literarische Bildung zielt vor allem auf die Entwicklung der literarischen Rezeptionskompetenz. Dazu gehört vor

3 Zum Verhältnis zwischen der Lesekompetenz und der literarischen Kompetenz im fremdsprachlichen Literaturunterricht vgl. u.a. BURWITZ-MELZER (2003, 2007) und JAZBEC (2009), im Bereich der muttersprachlichen Literatur z.B. HÄRLE / RANK (2004). 
allem die Fähigkeit, die Texte traditionsbewusst zu rezipieren, kulturell überlieferte Formen des Verstehens und Deutens anzuwenden und die ästhetische Codierung des Textes vor dem Hintergrund der kulturellen Epoche zu erkennen (literarische Konventionen, Stilmittel u. a.). ${ }^{4}$

Unter fremdsprachlichen Bedingungen hat die Beschäftigung mit Literatur analoge Aufgaben. Das übergeordnete Leitziel des Literaturunterrichts bleibt die Entwicklung der literarischen Kompetenz auf allen drei Ebenen. Das literarische Lernen erfolgt dank dem Kontakt mit dem fremdsprachlichen literarischen Text in und außerhalb des fremdsprachlichen Literaturunterrichts. Die literarische Bildung findet vor allem im Hochschulbereich statt, aber auch im Fremdsprachenunterricht an Grund- und Oberschulen, in dem literarische Texte behandelt werden.

In der gegenwärtigen Literaturdidaktik in Deutschland werden Leseförderung, literarische Bildung und Sprach- und Medienreflexion für drei grundlegende Aufgaben des Deutschunterrichts gehalten, die unter wechselseitiger Beachtung bewältigt werden sollen (ABRAHAM / KEPSER 2006:94). Diese Aufgaben bewahren ihre Aktualität auch unter fremdsprachlichen Bedingungen. Neben der Vermittlung des literarischen Gegenstandes soll im Literaturunterricht auch auf die Medienform der Literatur und die Entwicklung der Sprachkompetenz der Lerner geachtet werden.

\section{Ziele der fremdsprachlichen literarischen Bildung}

Aus den oben erläuterten Begriffen lassen sich Richtziele für die fremdsprachliche literarische Bildung im Hochschulbereich ableiten, die man tabellarisch folgendermaßen darstellen kann:

$4 \quad \mathrm{Zu}$ anderen Aspekten der literarischen Bildung, u.a. zum Geflecht Sprachkompetenz - Mehrsprachigkeit - Mulitkulturalität, vgl. HÄRLE / RANK (2008). 
Literarische Bildung in der Krise

Tab.1:

\begin{tabular}{|c|c|c|c|}
\hline \multirow[b]{2}{*}{ Kompetenzen } & \multicolumn{3}{|c|}{ Ziele der literarischen Bildung } \\
\hline & $\begin{array}{l}\text { Folgende Kenntnisse } \\
\text { sollen vermittelt } \\
\text { werden: }\end{array}$ & $\begin{array}{l}\text { 2. Psychomotorischer } \\
\text { (pragmatischer) } \\
\text { Bereich } \\
\text { Folgende Fertigkei- } \\
\text { ten sollen geschult } \\
\text { werden: }\end{array}$ & $\begin{array}{l}\text { 3. Affektiver } \\
\text { Bereich } \\
\text { Folgende Haltungen } \\
\text { sollen entwickelt } \\
\text { werden: }\end{array}$ \\
\hline $\begin{array}{l}\text { A. } \\
\text { Literarische Kompetenz } \\
\text { (auf den literarischen } \\
\text { Gegenstand bezogen) }\end{array}$ & $\begin{array}{l}>\text { Wissen über } \\
\text { historische } \\
\text { Entwicklungslinien } \\
\text { innerhalb der literari- } \\
\text { schen Epochen } \\
>\text { Wissen über den } \\
\text { Einfluss der histori- } \\
\text { schen Prozesse und } \\
\text { des geistigen Ideen- } \\
\text { guts auf die Formung } \\
\text { literarischer Strömun- } \\
\text { gen, ihre Normenset- } \\
\text { zung und Auffassung } \\
\text { von der Rolle der } \\
\text { Literatur in den } \\
\text { jeweiligen Epochen } \\
>\text { Kenntnis repräsen- } \\
\text { tativer Werke, ihrer } \\
\text { gattungs- und } \\
\text { epochenspezifischen } \\
\text { Merkmale } \\
>\text { Kenntnis ausge- } \\
\text { wählter Schriftsteller } \\
\text { und der Merkmale } \\
\text { ihres Schaffens unter } \\
\text { Einbeziehung } \\
\text { ihrer literaturge- } \\
\text { schichtlichen Re- } \\
\text { levanz } \\
>\text { Kenntnis ausge- } \\
\text { wählter Werke der } \\
\text { Kinder- und Jugendli- } \\
\text { teratur } \\
>\text { Wissen über } \\
\text { Unterschiede und } \\
\text { Ähnlichkeiten in } \\
\text { literarischen Entwick- } \\
\text { lungen in der }\end{array}$ & $\begin{array}{l}>\text { Benutzen von } \\
\text { Informationsquellen: } \\
\text { literarische Fachzeit- } \\
\text { schriften, Nachschla- } \\
\text { gewerke und Daten- } \\
\text { banken } \\
>\text { Umgang mit } \\
\text { Sekundärliteratur } \\
\text { (Interpretationen, } \\
\text { Literaturkritik) } \\
>\text { Wertungen aus- } \\
\text { drücken und begrün- } \\
\text { den } \\
>\text { Formanalyse der } \\
\text { literarischen Texte } \\
>\text { Erkennen der } \\
\text { Herkunft der Texte } \\
\text { (Entstehungszeit, } \\
\text { Autor) nach } \\
\text { epochentypischen } \\
\text { Merkmalen } \\
>\text { Unterscheidung } \\
\text { von Textsorten/ } \\
\text { Gattungen } \\
>\text { Selbstständige } \\
\text { Interpretation von } \\
\text { literarischen Texten } \\
>\text { Bezüge zu anderen } \\
\text { Texten und Medien } \\
\text { herstellen } \\
>\text { Bezüge zur eige- } \\
\text { nen Erfahrungswelt } \\
\text { und der gegenwärti- } \\
\text { gen Realität herstel- } \\
\text { len } \\
>\text { Umgang mit } \\
\text { anderen medialen } \\
\text { Formen der Literatur }\end{array}$ & $\begin{array}{l}>\text { Leselust } \\
>\text { Interesse an } \\
\text { deutschsprachiger } \\
\text { Literatur } \\
>\text { Bedürfnis, im } \\
\text { Erwachsenenleben } \\
\text { Kontakt zur } \\
\text { deutschsprachigen } \\
\text { Literatur zu erhalten } \\
>\text { Erweiterung des } \\
\text { geistigen Horizonts } \\
>\text { Kritikfähigkeit } \\
>\text { Genussfähigkeit } \\
>\text { Ästhetisches } \\
\text { Urteilsvermögen } \\
>\text { Literarhistori- } \\
\text { sches Bewusstsein }\end{array}$ \\
\hline
\end{tabular}


Ewa Turkowska

\begin{tabular}{|c|c|c|c|}
\hline & $\begin{array}{l}\text { polnischen und } \\
\text { deutschsprachigen } \\
\text { Literatur } \\
\text { > Kenntnis literari- } \\
\text { scher Gattungen } \\
\text { > Kenntnis aus- } \\
\text { gewählter } \\
\text { literaturwissen- } \\
\text { schaftlicher Theorien }\end{array}$ & $\begin{array}{l}\text { (Hörbuch, Hörspiel, } \\
\text { Film, digitale Litera- } \\
\text { tur) }\end{array}$ & \\
\hline $\begin{array}{l}\text { A. } 2 . \\
\text { Produktionskompetenz }\end{array}$ & $\begin{array}{l}>\text { Kenntnis der } \\
\text { Schreibnormen für } \\
\text { literarische Texte } \\
\text { (Gattungsmerkmale, } \\
\text { Schreibtechniken, } \\
\text { typische Themen und } \\
\text { Stilmittel) }\end{array}$ & $\begin{array}{l}>\text { Texte laut vortra- } \\
\text { gen } \\
>\text { Texte vorspielen } \\
\text { (szenische Darstel- } \\
\text { lung) } \\
>\text { Nacherzählen } \\
>\text { Texte in Form } \\
\text { einzelner literari- } \\
\text { scher Gattungen } \\
\text { schreiben } \\
>\text { Epochentypische } \\
\text { Schreibtechniken } \\
\text { verwenden (z. B. } \\
\text { innerer Monolog, } \\
\text { Sekundenstil) } \\
>\text { Medium für die } \\
\text { Gestaltung von } \\
\text { Literatur nach eige- } \\
\text { nen Bedürfnissen } \\
\text { wählen }\end{array}$ & $\begin{array}{l}>\text { Ästhetische } \\
\text { Genussfähigkeit in } \\
\text { Bezug auf die } \\
\text { Gestaltung von } \\
\text { Literatur }\end{array}$ \\
\hline $\begin{array}{l}\text { A. } 3 \text {. } \\
\text { Handlungskompetenz }\end{array}$ & $\begin{array}{l}\text { Kenntnis ausge- } \\
\text { wählter Gegenwarts- } \\
\text { autoren und ihrer } \\
\text { Werke }\end{array}$ & $\begin{array}{l}>\text { Literarische } \\
\text { Gespräche über } \\
\text { eigene Erfahrungen } \\
\text { und Wertungen } \\
\text { führen } \\
>\text { Literarische Texte } \\
\text { und Autoren nach } \\
\text { eigenem Geschmack } \\
\text { auswählen } \\
>\text { An Lesungen, } \\
\text { Autorentreffen } \\
\text { teilnehmen } \\
>\text { Buchkritiken, } \\
\text { Rezensionen lesen } \\
\text { und verfassen }\end{array}$ & $\begin{array}{l}\text { Individuellen } \\
\text { Lesegeschmack } \\
\text { entwickeln und } \\
\text { kritisch reflektieren }\end{array}$ \\
\hline
\end{tabular}


Neben der Entwicklung der literarischen Kompetenz werden im fremdsprachlichen literarischen Lernprozess auch Bezüge zur Entwicklung anderer Kompetenzen hergestellt. Dazu gehören:

Tab. 2:

\begin{tabular}{|c|c|c|c|}
\hline $\begin{array}{l}\text { B. } \\
\text { Fremdkulturelle } \\
\text { Kompetenz } \\
\text { (auf das Verstehen } \\
\text { der Kultur des } \\
\text { deutschsprachigen } \\
\text { Raumes bezogen) } \\
\text { entwickelt während } \\
\text { der literarischen } \\
\text { Bildung als Ergän- } \\
\text { zung zur Landes- } \\
\text { kunde/ } \\
\text { Kulturkunde }\end{array}$ & $\begin{array}{l}\text { Folgende Kenntnisse } \\
\text { sollen vermittelt werden: } \\
>\text { Wissen über historische } \\
\text { Hintergründe, geistige } \\
\text { Strömungen, Philosophie, } \\
\text { Architektur, bildende } \\
\text { Kunst in den deutsch- } \\
\text { sprachigen Ländern, die } \\
\text { in Verbindung zur Litera- } \\
\text { tur stehen } \\
>\text { Wissen über kulturelle } \\
\text { und gesellschaftspoliti- } \\
\text { sche Phänomene in den } \\
\text { Zielsprachenländern } \\
>\text { Wissen über Unter- } \\
\text { schiede und Ähnlich- } \\
\text { keiten in der kulturellen } \\
\text { und geistesgeschichtli- } \\
\text { chen Entwicklung des } \\
\text { eigenen Landes und der } \\
\text { Zielsprachenländer }\end{array}$ & $\begin{array}{l}\text { Folgende Fertigkei- } \\
\text { ten sollen geschult } \\
\text { werden: } \\
\text { > Erkennen der } \\
\text { Zugehörigkeit } \\
\text { literarischer Werke } \\
\text { zu einzelnen } \\
\text { kulturhistorischen } \\
\text { Epochen und } \\
\text { Strömungen }\end{array}$ & $\begin{array}{l}\text { Folgende Haltungen } \\
\text { sollen entwickelt } \\
\text { werden: } \\
>\text { Relativierung der } \\
\text { eigenen Wertvorstel- } \\
\text { lungen } \\
>\text { Verstehen des } \\
\text { Fremden/Anderen } \\
>\text { Abneigung gegen } \\
\text { Stereotype und Vor- } \\
\text { urteile } \\
>\text { Achtung vor } \\
\text { fremdkulturellen } \\
\text { Phänomenen und } \\
\text { Werten } \\
>\text { Toleranz }\end{array}$ \\
\hline $\begin{array}{l}\text { (auf die Beherr- } \\
\text { schung der deut- } \\
\text { schen Sprache } \\
\text { bezogen) } \\
\text { entwickelt während } \\
\text { der literarischen } \\
\text { Bildung als Ergän- } \\
\text { zung zur } \\
\text { Sprachpraxis }\end{array}$ & $\begin{array}{l}\text { Folgende Kenntnisse } \\
\text { sollen vermittelt werden: } \\
>\text { Ausgewählte } \\
\text { literaturwissenschaftliche } \\
\text { Begriffe } \\
>\text { Stilmittel der literari- } \\
\text { schen Sprache (Rhetorik) } \\
>\text { Wortschatz zur Be- } \\
\text { schreibung der eigenen } \\
\text { Leseeindrücke, emotio- } \\
\text { naler Zustände, Handlun- } \\
\text { gen und Haltungen der } \\
\text { Figuren }\end{array}$ & $\begin{array}{l}\text { Folgende Fertigkei- } \\
\text { ten sollen geschult } \\
\text { werden: } \\
>\text { Freies Sprechen, } \\
\text { u. a. Meinungsäu- } \\
\text { Berung, Diskussion, } \\
\text { Gespräch } \\
>\text { Kreatives } \\
\text { Schreiben } \\
>\text { Leseverstehen } \\
>\text { Wahrnehmung } \\
\text { der ästhetischen } \\
\text { Komponente der } \\
\text { deutschen Sprache }\end{array}$ & $\begin{array}{l}\text { Folgende Haltungen } \\
\text { sollen entwickelt } \\
\text { werden: } \\
>\text { Bereitschaft, sich } \\
\text { über Literatur zu } \\
\text { äußern }\end{array}$ \\
\hline
\end{tabular}


Aus den Tabellen wird ersichtlich, dass das literarische Lernen ein komplexer, lebenslanger Prozess ist. Nicht alle Ziele können an Hochschulen realisiert werden. Je nach Hochschultyp werden für den Literaturlehrgang diejenigen Ziele ausgewählt, die den allgemeinen Bildungszielen entsprechen.

\section{Didaktische Situation - Versuch einer Bestandsaufnahme}

Auf die gegenwärtige Praxis des Literaturunterrichts wirken sich vier Problembereiche negativ aus: die mangelhafte Sprachbeherrschung, Defizite in der literarischen Bildung an Oberschulen, veränderte lernpsychologische Dispositionen der Zielgruppen und die Marginalisierung der Literatur im Kulturbewusstsein der Gesellschaft. Das unzureichende Sprachniveau der Germanistikstudenten ist in Polen einerseits durch die Schulbildung, andererseits durch die demographische Entwicklung verursacht. Trotz (oder gerade wegen) der permanenten Reform des polnischen Schulwesens ist das Niveau des Fremdsprachenunterrichts an Grund- und Oberschulen immer noch nicht zufriedenstellend. Die zweite Fremdsprache ist eine Zeit lang aus der Liste der obligatorischen Fächer am Gymnasium verschwunden. Die schwierige ökonomische Situation der Schulen war daran schuld, dass infolgedessen die zweite Fremdsprache (d.h. oft das Deutsche) überhaupt nicht unterrichtet wurde. Die wenigen Schüler, die am Gymnasium drei Jahre Deutsch als erste Fremdsprache lernten, bekamen am Lyzeum oft keine Möglichkeit, den Deutschunterricht fortzusetzen, und mussten zum zweiten Mal mit der Stufe A1 beginnen. Das Stundenpensum für die Klassen mit erweitertem Deutschprogramm beträgt heute an vielen Lyzeen nur vier (und nicht wie früher sechs) Unterrichtsstunden in der Woche. Die veränderte Abiturprüfung mit vorwiegend geschlossenen Aufgaben bewirkt, dass im Fremdsprachenunterricht an Lyzeen nicht die Sprachkompetenz entwickelt wird, sondern die Fertigkeit, die ,Abiturprüfung zu bestehen'. Das demographische Tief verursacht, dass zum Studium auch Kandidaten zugelassen werden, die in der Abiturprüfung schwache Ergebnisse aufweisen. Seit einigen Jahren gibt es sogar an den renommiertesten polnischen Universitäten nach Ablauf des ersten Aufnahmetermins noch freie Studienplätze an mehreren Fakultäten. Im September-Termin werden dann auch schwächere Kandidaten aufgenommen, um die Gruppen aufzustocken. Im Germanistikstudium bewirken beide Faktoren, dass das Sprachniveau der Studenten des 1. Studienjahres so drastisch gesunken ist, dass es nach Meinung vieler Lehrender mit dem Niveau einer 2. Klasse des früher vier Jahre währenden Lyzeums verglichen werden kann. Hinzu kommen Defizite in der literarischen Bildung. Die humanistische 
Bildung an Lyzeen schrumpft in raschem Tempo und mit ihr der Polnischunterricht. Die Unkenntnis großer Werke der polnischen Literatur und der Weltliteratur, der literarischen Gattungen, der formellen Analyseverfahren, der Grundsätze der Poetik und Rhetorik nimmt zu. Ein knappes Stundenpensum und geringe Anforderungen in der Abiturprüfung bewirken, dass die polnische Literatur in der Schule nicht mehr vielseitig interpretiert und in ästhetischer Hinsicht genossen werden kann. Das Literaturwissen schöpfen die Schüler allzu oft nur aus Inhaltsangaben und fertigen Interpretationsklischees.

Die Folgen für den hochschulischen Literaturunterricht bleiben nicht aus. Die Lerner sind immer seltener imstande, eine Ganzschrift (Drama, Roman, längere Erzählung) in deutscher Sprache zu lesen und zu verstehen, den Inhalt anzugeben, in den literarhistorischen Kontext einzuordnen und die Thematik/Problematik zu erkennen. Da das Lesen des Originaltextes ihre Möglichkeiten immer öfter übersteigt, bedienen sie sich gern der Zusammenfassungen und Kommentare aus dem Internet. Die Arbeit am literarischen Text (Texterschließung, Interpretation) ist dadurch wesentlich erschwert. Die Lerner haben auch Schwierigkeiten, das literaturgeschichtliche und literaturtheoretische Wissen in der Zielsprache zu erwerben, Vorlesungen zu folgen und an Seminaren aktiv teilzunehmen. Die Unterrichtsarbeit dauert länger und ist weniger effektiv.

Andere Probleme im didaktischen Prozess betreffen veränderte lernpsychologische Voraussetzungen, die junge Lernergenerationen mitbringen: eine fragmentarische Wahrnehmung und ein zersplittertes Weltbild, Oberflächlichkeit, kein Bedarf an Systematisierung der Wissensbereiche und der Erschließung von Zusammenhängen, eine geringere Fähigkeit zum Zuordnen, Zusammenfassen, Übersichtlich-Machen und Systematisieren von Informationen. Das Gehirn der heutigen Schülergeneration, das einer ständigen Reizüberflutung (vor allem einer Bildüberflutung durch Fernsehen und Computer) ausgesetzt ist, geht auf eine andere Weise mit Informationen um. Um die Überlastung durch die Informations- und Bilderflut zu neutralisieren, verkürzt das Gehirn ihre Verarbeitung. Die Schüler eignen sich einzelne Informationen schneller an, verarbeiten sie aber sehr oberflächlich und können das erworbene Wissen nicht nutzen. Sie merken sich Informationen, können aber ihre Relevanz nicht einschätzen, Nebensächliches von Grundlegendem nicht unterscheiden, Gemeinsamkeiten oder Ursache-Wirkungs-Zusammenhänge nicht erkennen. Sie haben keine Konzentrationsfähigkeit: Sie widmen sich gerne vielen Sachen auf einmal, weil sie nicht imstande sind, eine komplexe Tätigkeit, die 
Aufmerksamkeit erfordert, zu Ende zu führen. (WIENER 2009:30f.) Heutige Studierende bringen auch neue Lerngewohnheiten mit: Sie sind an einen multimedialen Zugang zum Wissen und an interaktives Mitmachen gewöhnt. Die Beschäftigung mit dem Buch ist ihnen zu langweilig, der Leseprozess zu lang, die Komplexität einer Ganzschrift übersteigt ihr Fassungsvermögen.

Ein weiteres Problem betrifft die sinkende Rolle der Literatur im kulturellen Bewusstsein der Gesellschaft. Sozialisation und Enkulturation erfolgen gegenwärtig nicht mehr primär über Buch und (schöne) Literatur, sondern über Fernsehen und Multimedia. Die traditionellen Printmedien - nicht nur Bücher, sondern auch Zeitschriften und ehemals so beliebte Comics - sind in den Hintergrund getreten und dem Computerspiel, dem Internetsurfen, der häuslichen Nutzung von Standard- und Übungssoftware (und nach wie vor dem Film und Fernsehen) gewichen. Die Ergebnisse der empirischen Leseforschung zeigen, dass das Bedürfnis nach Unterhaltung, nach Fiktionalität, nach dem ,Eintauchen' in wirklichkeitsferne Welten und nach Identifikation mit fiktiven Figuren in erster Linie von den AV- und Multimedien gedeckt wird. Die jungen Leute berichten über ihre Identifikationserlebnisse nur in Verbindung mit Hörspielkassetten, Filmen, interaktiven Computerspielen (MUDs, Ego-Shooter) und Fernsehprogrammen (Affekt-TV, Reality-TV, Gewinnspiele). Von der Buchlektüre erwarten die jungen Leser vor allem ,Bildung ' und Informationen in kognitiver Hinsicht, also eine Wissensakkumulation. (DAWIDOWSKI 2009:348)

Literatur ist heute immer öfter Medienverbundliteratur; die kulturelle Praxis also, in die Heranwachsende einzuführen sind, ist eine Praxis des Umgangs mit allen ästhetikfähigen Medien. Aus dieser pädagogischen Situation ergeben sich Konsequenzen für den Literaturunterricht. Ein Literaturunterricht, der die Voraussetzungen der Lernergruppen ernst nimmt, sollte neben der traditionellen literaturwissenschaftlichen Beschäftigung mit Literatur die Sprachschwierigkeiten der Lerner beim Kontakt mit dem fremdsprachlichen literarischen Lernstoff minimalisieren und sie beim Verstehen der Vorträge und Lektüren unterstützen, die Kenntnis und Vorliebe der Lerner für die neuen Medien zur produktiven, kreativen Unterrichtsarbeit einsetzen, den ästhetischen Genuss aus dem Kontakt mit Literatur vergegenwärtigen und auf die unersetzliche Rolle der Literatur im gesellschaftlichen Leben und in der individuellen Entwicklung hinweisen, damit die Literatur aus dem kulturellen Bewusstsein der jungen Generation nicht verschwindet. 


\section{Situation des Faches}

In der globalisierten Welt haben auch Krisen einen globalen Charakter. Von einer Krise der Germanistik wird sowohl in Deutschland als auch in Polen, Rumänien, Ungarn, aber auch im angloamerikanischen Sprachraum, in Brasilien und Korea gesprochen, wobei man ähnliche (zum Teil im 3. Abschnitt geschilderte) Schwierigkeiten beklagt. ${ }^{5}$ Allgemeine Tendenzen zeigen sich in der Öffnung der traditionellen philologischen Germanistik, in interdisziplinären Studiengängen, in der Hinwendung zur Kultur- und Kommunikationswissenschaft und in der interkulturellen Germanistik, in der stärkeren Bindung an die Anforderungen des Arbeitsmarktes, in der Spezialisierung und Hinwendung zu digitalen Medien in Forschung (,Digital Humanities', ,Computerphilologie') und Lehre. Bachelor- und Masterstudiengänge profilieren sich. An Technischen Hochschulen und Berufshochschulen (PWSZ) entstehen neuphilologische Institute, an denen die Germanistik mit Marktwirtschaft, Management, Kommunikationswissenschaft bzw. Tourismus gekoppelt wird. ${ }^{6}$ Literaturkurse dauern ca. 150 Stunden und bilden nur einen kleinen Teil der Gesamtausbildung. Diese Entwicklung zeigt, dass die traditionellen philologischen Fächer nicht mehr das A und $\mathrm{O}$ des Germanistikstudiums bilden. Literatur verliert im gesellschaftlichen Kommunikationssystem und im alltäglichen Leben an Bedeutung. Auch innerhalb der Literaturwissenschaft vollziehen sich Veränderungen. Unter Literaturwissenschaftlern herrscht heutzutage Konsens darüber, dass die Literaturwissenschaft in der Informations- und Mediengesellschaft ihr Profil und ihre Funktion verändern muss.

5 In den mitteleuropäischen Ländern wird die Krise der Germanistik intensiv diskutiert. In Rumänien, an der Universität Partium in Oradea, fand im September 2012 eine Tagung zum Thema „Krisen als Wendepunkte in der Germanistik" statt.

6 Ähnlich in Deutschland: In Bayreuth gibt es Bachelor-Module in Jura und BWL; die Universität Bamberg bietet zusätzliche Seminare für Verlags- und Urheberrecht an, um das germanistische Studium marktorientierter zu gestalten (FRESE 2011). Es werden Master-Studiengänge angeboten, die Spezialisten für konkrete Berufe ausbilden, z. B. die ,Interkulturelle Germanistik Deutschland - China ' an der Universität Göttingen und an zwei chinesischen Partnerhochschulen, deren Absolventen als Kulturvermittler für den Arbeitsmarkt fungieren. Besonders praxisorientiert ist auch der Studiengang ,Deutsch als Fremdsprache‘, der heute an mehr als fünfzig deutschen Hochschulen angeboten wird und sich einer nicht nachlassenden Popularität erfreut (JosT 2010). Die amerikanischen ,German Studies' werden interdisziplinär, beziehen die deutsche Alltagskultur mit in die Ausrichtung des Faches ein (AlLROGGEN 2006). 
Die Literatur und ihre Geschichte werden nicht mehr unangefochten im Zentrum des Faches stehen können (VoGT 2008:19f.). ${ }^{7}$

Diese Entwicklung kann man bedauern, aber man muss sich ihr anpassen. Sie eröffnet für die literarische Bildung grundlegende Fragen, die bildungspolitische, didaktische und literaturwissenschaftliche Aspekte gleichermaßen betreffen: Nach welchen Grundsätzen sollte ein Mini-Literaturlehrgang im Bachelor-Studium gestaltet werden? Was sollte zum Ausgangspunkt und Leitfaden genommen werden: die Bedürfnisse der Literaturwissenschaft, der Zielgruppen oder die des Arbeitsmarktes? Sollte man die ,Wissenschaftlichkeit' des Literaturlehrgangs bewahren oder die Beschäftigung mit Literatur praxisnah nach den Bedürfnissen des künftigen Berufs ausrichten? Wie viel Literaturwissenschaft braucht ein angehender Deutschlehrer, Manager, Jurist, Reiseführer? Sollte man literarische Texte nur auf literaturwissenschaftliche Art analysieren und interpretieren, oder darf man sie ,instrumentalisieren und zur Sprachbildung einsetzen? Lassen sich diese Auffassungen in Einklang bringen oder schließen sie einander aus?

\section{Integrierter Literatur- und Sprachunterricht}

Seit Mitte der 1990er Jahre funktioniert in der berufsorientierten Hochschulausbildung ein didaktisches Konzept, das nicht die einzelnen philologischen Disziplinen wie Literatur- oder Sprachwissenschaft, sondern die Voraussetzungen der Zielgruppen und die Anforderungen des Arbeitsmarktes zum Leitfaden der Ausbildung macht. ${ }^{8}$ Davon ist auch die Konzeption der berufsorientierten literarischen Bildung beeinflusst. Die in diesem Abschnitt präsentierte Form des Literaturlehrgangs beschreibt die Realisierung dieser Konzeption in der Unterrichtspraxis am Fremdsprachenkolleg in Radom, wo die Richtlinien dieser Konzeption aufgrund der Beteiligung von vier Lehrpersonen an ihrer Erarbeitung aufs Genaueste befolgt werden. Die Arbeit am literaturgeschichtlichen und literaturtheoretischen Stoff erfolgt nicht in der traditionellen Form des Vortrags, in dem das Wissen von der Lehrperson ausschließlich akustisch-verbal dargeboten wird und die Lerner zuhören und sich Notizen machen. Alle Vorträge werden mit visuellen Begleitmaterialien

7 Ein solcher Wandel ist nicht einmalig: Die Klassische Philologie, die im 19. Jhd. im Zentrum der humanistischen Bildung stand, verlor unter dem Druck massiver Modernisierung der Gesellschaft und durch die Durchsetzung der Nationalsprachen ihre führende Position und wurde zum ,Orchideenfach“ (VOGT 2008:20).

$8 \quad$ Es entstand für die berufsorientierte Deutschlehrerausbildung (STASIAK 1995). 
versehen, um die Informationsentnahme zu erleichtern. Dazu gehören sowohl statische Visualisierungsmittel wie Bilder, Schemata, Fotos, Poster, Collagen als auch audiovisuelle Materialien (didaktische Filme, Aufnahmen von Theateraufführungen) und digitale Medien: Power-Point-Präsentationen, Software, literaturwissenschaftliche Lexika online, audiovisuelle Materialien aus YouTube (wie z. B. die Sendereihen Lauter schwierige Patienten mit Marcel Reich-Ranicki und Lyrik für alle, in der der Schauspieler Lutz Görner Gedichte rezitiert und Informationen über ihre Autoren vermittelt, Dokumentarfilme über Schriftsteller, Romanverfilmungen u. a.). Sowohl die Vorträge der Lehrperson als auch die audiovisuellen Materialien werden stets mit Begleitübungen versehen, die das Hörverstehen bzw. Leseverstehen fördern und die Informationsverarbeitung fordern. Als Literaturübungen sind alle aus der Didaktik/Methodik des DaF-Unterrichts bekannten Übungstypen gut geeignet, z. B. Lückenübungen, Zuordnungsübungen, Fehler- und Wortsuche, Richtig-Falsch-Übungen. ${ }^{9}$ Dank dem Einsatz der Übungen wird der Lernstoff sprachlich vorentlastet, die Informationen werden effektiver im Gedächtnis gespeichert. Die Arbeit am literarischen Lernstoff und die Spracharbeit ergänzen und unterstützen einander. Als Beispiel folgt eine HV-Begleitübung zum Film Tränen des Vaterlands von Inter Nationes (1999).

Übung 3. Ergänzen Sie die Lücken: (00 bis 02 Min.)

Barock - das ist das Jahrhundert zwischen Reformation und 1)......................................: unsere frühe Neuzeit. In dieser Epoche findet die letzte Auseinandersetzung zwischen 2)........................................ und 3)............................................. statt. Die Menschen erleben die extremen Gegensätze zwischen den Zerstörungen des 4)..................................... Krieges, den Schrecken der 5)............................ und der Prachtentfaltung des 6)...................................... . Deutschland ist 7)............................... in kleinere und größere Fürstentümer. Die Macht des Kaisers ist 8).................................., und es gibt kein 9).........................und 10)........................ Zentrum, keine Hauptstadt. Geschrieben wird 11)................................... Deutsch ist die Sprache der 12)...

Stände, eine Sprache ohne Selbstbewusstsein. Dass Deutsch eine 13) ...................................... sein kann - diesen Beweis mussten Barock-Autoren wie 14).................................. erst einmal liefern. Sie ahmten Vorbilder aus der 15) ......................................... und dem 16).................................. nach

9 Übungen zur Literaturgeschichte befinden sich auf der Website http:// literaturdidaktik.republika.pl/elearning.html (20.11.2011) sowie bei TURKOwSKA (2006:233-247). 
und rechtfertigten durch strenge 17).

den Gebrauch des Deutschen. ${ }^{10}$

Eine andere Übung zum Film Das Land der Griechen mit der Seele suchend... Die Weimarer Klassik (BAUER 1996) verbindet die Entwicklung des Hörverstehens mit der Erweiterung des literaturgeschichtlichen Wissens. Die Lerner bekommen Arbeitsblätter mit sieben Kurztexten über Weimar zu Goethes Lebzeiten, von denen drei im Film fehlen, aber zusätzliche wichtige Informationen vermitteln. Die Lerner müssen entscheiden, welche Information sie im Film gehört haben. Die Vorträge der Lehrperson werden von ähnlichen Übungen begleitet. Statt Notizen zu machen, lösen die Studierenden die Übungen während des Vortrags. Die Lösungen werden im Plenum überprüft.

Große Schwierigkeiten bereitet den Lernern der Kurs ,Einführung in die Literaturwissenschaft ', der vielerorts traditionell im ersten Semester als separate Lehrveranstaltung dargeboten wird. Wegen der großen Sprachschwierigkeiten der Lerner wird dieser Kurs heutzutage manchmal auf Polnisch durchgeführt. Um das zu vermeiden, wurde der separate Lehrgang aufgelöst, seine Lerninhalte wurden auf alle drei Jahre des Bachelor-Studiums verteilt. Der Literaturlehrgang ist als ein einheitlicher Kurs konzipiert, ohne die Aufteilung in Literaturgeschichte und Literaturtheorie. Diese Kursform macht es möglich, im Rahmen einer Lehrveranstaltung Literaturtheorie, Literaturgeschichte und Textinterpretation zu verbinden und so die theoretischen Grundsätze mit der praktischen Interpretationsarbeit zu verknüpfen. Die Aufhebung der separaten Lehrgänge für Literaturtheorie, Literaturgeschichte und Textinterpretation erlaubt es, die eigentlichen Zusammenhänge zwischen den einzelnen Bereichen der Literaturwissenschaft wieder herzustellen und bei den Lernern ein kohärentes Bild der literarischen Entwicklung entstehen zu lassen. Auf diese Weise kann man literaturtheoretische Themen an Beispielen von Pflichtlektüren ausführlich behandeln und ihre praktische Rolle bei der Werkinterpretation aufzeigen. Alle behandelten Fachtexte zu literaturtheoretischen Themen wie z.B. Rezeptionsästhetik, Gender und Queer Studies, Dekonstruktion, Hermeneutik werden als Leseverstehensübungen didaktisiert. Es folgt ein Beispiel für eine LV-Übung zu einem Fachtext über Rhetorik:

10 Lösung: 1) Aufklärung 2) Reformation 3) Gegenreformation 4) Dreißigjährigen 5) Pest 6) Absolutismus 7) zersplittert 8) begrenzt 9) politisches 10) kulturelles 11) lateinisch 12) einfachen 13) Literatursprache 14) Gryphius 15) Antike 16) Ausland 17) Regeln. 
Übung 1. Finden Sie Fehler in den folgenden Informationen und korrigieren Sie sie: $[\ldots]$

5. Die Rhetorik war ursprünglich ästhetisch orientiert. Mit dem nach rhetorischen Regeln ausgeführten Text hatte der Redner zum Ziel, die Rezipienten des Textes zu einer bestimmten Überzeugung zu bringen.

6. Für die Literaturwissenschaft ist vor allem das dritte Produktionsstadium des Textes, die ,dispositio', von Bedeutung.

7. Von den vier Prinzipien der ,elocutio' ist die ,latinitas', d.h. die sprachliche Korrektheit, für die ästhetische Qualität der Sprache relevant.

8. Tropen sind Formen der uneigentlichen Rede, übertragene Ausdrücke. Sie entstehen durch Verschiebungen auf der syntaktischen Ebene.

9. Rhetorische Figuren entstehen durch Veränderungen auf der Bildebene. Hier geht es nicht um den eigentlichen oder uneigentlichen Sinn, sondern um die Anordnung der Wörter im Satz. ${ }^{11}$

Eine besondere Bedeutung hat die lernerorientierte Arbeit an literarischen Werken. Den Lernenden werden vor und während der Hauslektüre Hilfen angeboten, die sie auf den Kontakt mit einem komplexen Literaturwerk vorbereiten, es sprachlich und inhaltlich vorentlasten und die Verstehensschwierigkeiten abbauen. Vor der Behandlung eines literarischen Werkes im Seminar wird in der vorangehenden Unterrichtsstunde eine vielseitige Vorentlastungs- und Einführungsphase durchgeführt. Neben dem ,informierenden Einstieg' (Informationen zum literaturgeschichtlichen Kontext, Entstehungsgeschichte, Stoff usw.) wird großer Wert auf den , atmosphärischen Einstieg ‘ gelegt. Die Lerner antizipieren den Inhalt, erzählen oder schreiben Geschichten zu Filmtrailern (zu finden auf YouTube), einzelnen Szenen, ausgewählten Zitaten oder bilden Geschichten, in denen die Figuren aus dem zu lesenden Werk auftreten. Auf diese Weise machen sie sich mit der Fabel vertraut, was den Verstehensprozess erleichtert. Die Hauslektüre wird von ,Aufgaben

11 Lösung: 5. Die Rhetorik war ursprünglich praxisorientiert. Mit dem nach rhetorischen Regeln ausgeführten Text hatte der Redner zum Ziel, die Rezipienten des Textes zu einer bestimmten Überzeugung zu bringen. 6. Für die Literaturwissenschaft ist vor allem das dritte Produktionsstadium des Textes, die ,elocutio', von Bedeutung. 7. Von den vier Prinzipien der ,elocutio" ist der ,ornatus', d.h. die sprachliche Ausschmückung der Rede, für die ästhetische Qualität der Sprache relevant. 8. Tropen sind Formen der uneigentlichen Rede, übertragene Ausdrücke. Sie entstehen durch Verschiebungen auf der semantischen Ebene. 9. Rhetorische Figuren entstehen durch Veränderungen auf der Satzebene. Hier geht es nicht um den eigentlichen oder uneigentlichen Sinn, sondern um die Anordnung der Wörter im Satz. (Die Übung TuRKowsKA 2011:148, die Lösung:271). 
während des Lesens' begleitet, sie beziehen sich auf den Inhalt und die wichtigsten Textstellen. Die Lerner bekommen vor dem Lesen den Fragebogen zur Textinterpretation, anhand dessen die Interpretation im darauffolgenden Unterricht verläuft. Die Orientierung über die kommenden Interpretationsaufgaben steuert die Informationsentnahme und erleichtert das Verstehen. Die traditionelle Textanalyse und Interpretation wird um handlungsund produktionsorientierte Aktivitäten ergänzt. Dies erlaubt, neben der Rezeptionskompetenz die literarische Produktionskompetenz zu entwickeln. Außer der traditionellen Interpretation werden ,weiterführende Aufgaben durchgeführt. Die Lerner schreiben Texte im Sekundenstil, innere Monologe, Rezensionen, führen Interviews mit Handlungsfiguren durch, bauen Standbilder, führen Rollen- und Planspiele durch, gestalten Diskussionen. Der Literaturkurs wird durch die Verwendung literaturbezogener Lerninhalte in anderen Lehrgängen erweitert: in der Kulturkunde, Landeskunde, Geschichte und vor allem in der Sprachpraxis (im Leseverstehen, Hörverstehen und in dem Teilfach ,Integrierte Sprachfertigkeiten '). Im fächerübergreifenden Unterricht wird sehr oft mit literarischen Texten bzw. mit literaturwissenschaftlichen Fachtexten gearbeitet. Im Fach ,Hörverstehen' z. B. werden HVÜbungen zu Gedichten deutscher Klassiker (im Programm Lyrik für alle), zu romantischen Liedern, Opern (z.B. Mozarts Zauberflöte), zu Hörspielen, Audiobüchern, Videoaufnahmen von Theateraufführungen und zu Romanverfilmungen eingesetzt.

Um einen Bezug zur Berufspraxis der zukünftigen Lehrer herzustellen, wird der Literaturdidaktik im Rahmen des Literaturlehrgangs ein Semester (30 Stunden) gewidmet. Es werden Besonderheiten des literarischen Lesens und ihre Konsequenzen für den didaktischen Prozess, die Ziele, Inhalte und Methoden der Arbeit mit epischen und lyrischen Texten im Deutschunterricht ausführlich besprochen, Unterrichtsentwürfe geschrieben und präsentiert. Nach der Einführung des Curriculums von STASIAK (1995) war eine Auswertung des Konzeptes nach drei Jahren Erprobung geplant, die Auswertung fand aber nicht statt, und es gibt auch keine Untersuchungen, ob das Curriculum tatsächlich in die Unterrichtspraxis umgesetzt wurde. Wegen fehlender Untersuchungen und Auswertungen dieses berufsorientierten Konzeptes kann man heutzutage nicht eindeutig feststellen, in welchem Ausmaß es die Ausbildungspraxis $\mathrm{zu}$ beeinflussen vermochte. Die Diskussionen auf den Tagungen des Goethe-Instituts in der zweiten Hälfte der 1990er Jahre zeigten, dass dieses Konzept wichtige Impulse für die Erarbeitung neuer Literaturprogramme an Fremdsprachenkollegs lieferte. Auf denselben Tagungen wurde es aber auch aufgrund seiner mangelnden literaturwissenschaftlichen 
Ausrichtung heftig kritisiert. ${ }^{12}$ Heutzutage scheint in allen Bildungsinstitutionen die Zeit gekommen zu sein, in dem die reine Theorie an Wirkungsmacht verloren hat, die Praxisorientierung wird großgeschrieben. Das macht dieses Konzept wieder beachtenswert.

\section{Literarische Bildung online}

Die Entwicklung der neuen Medien bietet neue, bisher unbekannte Möglichkeiten der Weiterführung und Ergänzung der literarischen Bildung online. Ein besonders großes Lernpotential steckt im E-Learning. Germanistische Institute in unserem Zielsprachenland und auf der ganzen Welt bieten heutzutage interessante Lernprogramme online an. ${ }^{13}$ Viele polnische Institute greifen auf diese Möglichkeit zurück. In Polen werden vorwiegend MoodleLernplattformen eingesetzt, die es erlauben, den Studierenden Ergänzungsmaterialien anzubieten, zusätzliche Lernaufgaben zu stellen, ihre selbstständige Arbeit individuell zu betreuen und die Lernergebnisse zu kontrollieren. Moodle hat allerdings den Nachteil, dass nur die Studenten der jeweiligen Hochschule an den Kursen teilnehmen dürfen. Den Gästen, die über kein Passwort verfügen, bleibt die Möglichkeit der Wissensergänzung vorenthalten. Im Ausbau der Lernplattformen zu allen germanistischen Studienfächern (neben Literaturwissenschaft auch Sprachwissenschaft, Geschichte, Landeskunde, Didaktik/Methodik DaF, Sprachpraxis) und ihrem systematischen Einsatz liegt eine große Chance zur Ergänzung und Erweiterung der Unterrichtsarbeit. Dem effektiveren Behalten von Informationen dient auch die selbstständige Arbeit der Studenten mit Online-Lexika und mit der Software zur deutschen Literatur. ${ }^{14}$ Einen Teil der Wissensvermittlung und der handlungsorientierten Interpretation kann man heute ins Internet verlegen, nämlich mit Studierenden über eine eigene Webseite, über Mailinglisten und Blogs kommunizieren oder Internet-Projekte zur Literatur erstellen. Es ist besonders hoch zu schätzen, dass das Internet eine interessante Arbeit an der Entwicklung der produktiven Literaturkompetenz (Schreiben und

$12 \mathrm{Zu}$ den Kontroversen um das Teilcurriculum Literatur vgl. TURKOWSKA (2000).

13 Z. B. das Programm der Universität Kiel auf der Website http://www. literaturwissenschaft-online.uni-kiel.de/e-learning/barock.asp (20.11.2011), daneben gibt es zahlreiche Unterrichtsmaterialien wie Folien, Online-Vorträge.

14 Einen Überblick über das aktuelle Software-Angebot gibt es u. a. auf der Website http://www.amazon.de/gp/bestsellers/software/408274/ref=pd_zg_hrsr_ sw_3_4_last zugänglich (20.11.2011). 
multimediales Gestalten von Texten) und der Handlungskompetenz (Teilnahme am literarischen Leben des Zielsprachenlandes) ermöglicht. Die Interaktivität und multimediale Attraktivität der Computermedien strahlen auf die heutige Lernergeneration eine große Faszination aus, die eine sehr wichtige Motivationsfunktion hat. Es ist daher sehr wichtig, die unbegrenzten Möglichkeiten des Internets für den Literaturunterricht zu nutzen.

Der Einsatz der neuen Medien trägt zur Realisierung eines weiteren wichtigen Lernziels bei - zur Entwicklung der Medienkompetenz. Eine umfassende Medienkompetenz bildet die Voraussetzung für die Existenz in der modernen Informationsgesellschaft. Die Befähigung der Schüler zu einer effektiven Informationsentnahme, aber auch zu einem kritischen Umgang mit Medien halten die Didaktiker deswegen für eine wichtige Bildungsaufgabe. Das Erreichen der Medienkompetenz ist ein wichtiges Ziel der heutigen Pädagogik und Didaktik. Die Medienkompetenz ist seit Beginn der 1970er Jahre ein Schlüsselbegriff in der Medienpädagogik. Zur Begriffsbestimmung hat DIETER BAACKE (1997) den Grundbaustein gelegt. ${ }^{15}$ Allgemein versteht man darunter die Fähigkeit, sich in der Medienwelt zurechtzufinden: mediale Kommunikate und ihre spezifischen Zeichen zu verstehen, sie kritisch zu bewerten und Informationen verarbeiten zu können. Es wird dabei betont, dass der Mensch den Medien nicht willenlos ausgeliefert sein, sondern als autonomer Mediennutzer mit Medien souverän umgehen soll. Die Medienkompetenz wird als ein Teil der kommunikativen Kompetenz des Menschen angesehen. Medienkompetenz und Literatur stehen in enger Beziehung zueinander. Literatur ist heute immer öfter Medienverbundliteratur, d.h., dass die kulturelle Praxis, in die Heranwachsende einzuführen sind, die Praxis des Umgangs mit allen ästhetikfähigen Medien ist. Eine besondere Beachtung unter Literaturdidaktikern verdient heutzutage das Problemfeld ,Computermedien und Literatur(unterricht)', das unter zahlreichen Aspekten intensiv

15 Zur Medienkompetenz gehören nach Baacke die Medienkritik, also die Fähigkeit, sich analytisch, ethisch und reflexiv auf die Medien zu beziehen, die Medienkunde - das Wissen über Medien, die Fähigkeit zur Medienrezeption und zu aktiver Mediengestaltung (nach Moser 2010:218). Andere Konzepte stammen von Aufenanger, Tulodziecki, Kübler, Groeben; vgl. die kompakte Übersicht bei SÜSS / LAMPERT / WiJNEN (2010:106-120). Verschiedene Autoren bezeichnen die Dimensionen der Medienkompetenz unterschiedlich. Hurrelmann benennt folgende Kompetenzebenen: das Medienwissen/Medienbewusstsein, medienspezifische Rezeptionsmuster, die medienbezogene Genussfähigkeit, die Selektion und Kombination von Mediennutzung, produktive Partizipationsmuster, Anschlusskommunikationen. (HURRELMANN / BECKER 2003:16) 
erforscht wird (z.B. BICKENBACH / MAYE 2009; SEGEBERG / WinKo 2005; HURRELMANN / BECKER 2003 und viele mehr). ${ }^{16}$

Im weiteren Teil des Artikels wird zwei Formen der literaturbezogenen Online-Aktivität der Lerner Aufmerksamkeit geschenkt, die effektiv zur literarischen Bildung beitragen: der kollektiven Schreibarbeit an Hyperfiktionen und Blogs. Die im Abschnitt 6.1. und 6.2. geschilderten Projekte wurden am Fremdsprachenkolleg in Radom durchgeführt.

\subsection{Hyperfiktionen}

Die Hyperfiktion ist eine neue Form der postmodernen Erzählliteratur und als solche für den Literaturlehrgang von Bedeutung. Sie realisiert visuell und haptisch die in der ganzen Erzählliteratur des 20. Jhd.s präsente Multilinearität und Multiperspektivität der Narration.

Die Hyperfiktionen bieten vielfältige Möglichkeiten, mit dem literarischen Text kreativ und handlungsorientiert zu arbeiten. Ihre offene, mosaikartige Struktur und eine Vielzahl von Leerstellen laden zur Ergänzung um Eingreiftexte, zum Um- und Weiterschreiben an. Das strenge Prinzip der Kausalität ist aufgehoben, jeder Textausschnitt kann praktisch mit jedem anderen verlinkt werden, deswegen können die Lerner beim Ergänzen ihrer Phantasie freien Lauf lassen.

Die Form der Hyperfiktion diente als Vorlage für ein didaktisches Projekt, das mehrere Kompetenzen und Fertigkeiten verbindet - Elisa 2.0. ${ }^{17}$ Die Idee für die Fabel wurde der Novelle Fräulein Else von A. Schnitzler entnommen. Als Vorlage für eine Hyperfiktion hat sie mehrere Vorteile: einen entsprechenden Umfang, einen dramatischen Handlungsverlauf, interessante Figuren, eine aktuelle Thematik. Einen besonderen Vorteil stellt die Erzähltechnik des inneren Monologs dar. Für diese Form der Gedankenwiedergabe sind freie Assoziationen, die ungebundene Reihung von Aussagen und der sprunghafte Themenwechsel charakteristisch. Die lockeren inhaltlichen und syntaktischen

16 In diesem Artikel verzichte ich aufgrund des begrenzten Seitenumfangs bewusst auf die Einführung und nähere Erläuterung des komplexen Begriffs , digitale Literatur' wie auch auf die Schilderung der theoretischen und literaturdidaktischen Aspekte von Hyperfiktionen und Blogs. Vielfältige Formen des literarischen Schreibens im Netz besprechen z.B. FEHR / GOND $(2002,2003)$ und BOESKEN (2010). Zur Hyperfiktion vgl. z. B. SimANOwSKI (2002) und PIESTRAK-DEMIREZEN (2009), eine Übersicht bietet MAHNE (2007:110-125).

17 http://elisa20.republika.pl (20.11.2011). 
Zusammenhänge erlauben das Einmontieren von mehreren Inhalten in die Gedankengänge der Figuren und die Ergänzung des Originaltextes um neue Handlungsepisoden. Neben der originalen Handlung, die in der vereinfachten Form nachgestaltet wird, befinden sich in Elisa 2.0 neue, von den Studenten ausgedachte Episoden, in denen andere Handlungsfiguren dargestellt werden und die Handlung ausgebaut wird. Die von den Lernern verfassten Eingreiftexte haben ebenso die Form des inneren Monologs. Die Autor(inn)en versuchen, das Innenleben der Figuren und das äußere Geschehen mit den Mitteln des inneren Monologs überzeugend darzustellen. Die fiktionale Ebene wird um die von den Studenten verfassten Sachtexte bereichert, die sich auf die in den Gedankengängen der Figuren angedeuteten Sachverhalte beziehen. $\mathrm{Zu}$ diesem Zweck werden entweder Andeutungen aus dem Originaltext ausgewählt (Drogen, Kunsthandel, Psychoanalyse) oder neue Begriffe in den Text einmontiert, die Anlass für die Recherche über wichtige literarische oder landeskundliche Problemfelder sind (der innere Monolog, Singles, Spielsucht, Drogensucht, Drogenvisionen in Literatur und Musik). Weitere Links führen von der Sachtextebene nach außen, in das Docuversum des WWW. Die Grenze zwischen literarischen Texten und Sachtexten wird auf diese Weise aufgehoben. Neben diesen beiden befinden sich hier auch Filmausschnitte, eigens gedrehte Videos von Student(inn)en (in Entstehung) und Lieder. Sie machen Elisa 2.0 zu einem hypermedialen Gebilde, das die Struktur des WWW nachahmt.

Das Projekt verfolgte mehrere sprachbezogene, literaturbezogene, landeskundliche und didaktische Ziele: die Erweiterung des Literaturwissens, die Entwicklung des kreativen Schreibens, die Einübung der Schreibtechnik ,innerer Monolog', die selbstständige Informationsentnahme und die Strukturierung von Informationen über ausgewählte landeskundliche und literarische Themen, die Förderung der Lernerautonomie und Kreativität, die Lieferung von Beispielen für den Einsatz der handlungsorientierten Unterrichtsmethoden und der neuen Medien im Deutschunterricht. Das Projekt Elisa 2.0 wurde mit den Studenten des 2. Jahres am Fremdsprachenkolleg in Radom durchgeführt und dauerte zwei Semester. Die Arbeit auf Fiktions- und Sachtextebene wurde kurz vor der Beschäftigung mit Fräulein Else im Literaturunterricht beendet. Die Textinterpretation im Unterricht verlief sehr schnell und brachte sehr gute Ergebnisse. Die Student(inn)en haben während der Arbeit an den einzelnen Lexien der Hyperfiktion eine ausgezeichnete Kenntnis des Novelleninhalts, der Figuren und der Schreibtechnik des inneren Monologs erworben. Die Konnotationen zur Psychoanalyse, die sonst schwer nachzuvollziehen sind, waren für die Student(inn)en nun verständlich. Nach dem Unter- 
richt wurde der Elisa-Hypertext um die Textinterpretationen der Studierenden, um die Informationen über den Autor und die Wiener Moderne ergänzt. Das Projekt erweiterte literarisches und landeskundliches Wissen der Lerner und ihr sprachliches Können, entwickelte die ästhetische Genussfähigkeit und machte viel Spaß. Die Arbeit an der Hyperfiktion ist allerdings sehr zeit- und arbeitsaufwendig, sowohl für die Lerner als auch für die Lehrer. Notwendig sind Basiskenntnisse in der Benutzung eines Programms zur Gestaltung von Webseiten seitens der Lehrperson. Das alles macht das kollektive Schreiben an einer Hyperfiktion zu einem anspruchsvollen und daher selten eingesetzten Unterrichtsprojekt. ${ }^{18}$

\subsection{Weblogs}

Die Blogs entstehen dank dem individuellen oder gemeinsamen Schreiben im Netz. In den Blogs werden sowohl literarische Texte als auch persönliche Reflexionen der Autor(inn)en, Erlebnisse, Ratschläge oder Kommentare zu aktuellen Ereignissen veröffentlicht. Die Literaturblogs haben unterschiedliche Formen. Sie beinhalten eigene literarische Texte ihrer Autor(inn)en (Kurzprosa, Lyrik), Texte anderer Autoren, z. B. Lieblingsgedichte des Blogautors, eigene Fotos usw. Die Einsatzmöglichkeiten der Blogs im Literaturunterricht sind daher sehr vielfältig. ${ }^{19}$ Man kann sie zur Vertiefung und Ergänzung der Arbeit an Lerninhalten verwenden, z.B. zum Recherchieren nach zusätzlichen Informationen oder zur Weiterführung der Interpretationsarbeit. Ein Beispiel für ein didaktisches Literaturblog ist LiteraBLOGtur, ein Projekt für den Literaturunterricht am Fremdsprachenkolleg in Radom. ${ }^{20}$ Das Blog wurde zu

18 Die Evaluation des Projekts wurde online durchgeführt, die Meinungen der Autor(inn)en und Leser(inn)en zur Entstehung und zum Endeffekt können im LiteraBLOGtur auf der Website http://literablogtur.blog.onet.pl (20.11.2011) nachgelesen werden.

19 Zum Einsatz von Blogs im Literatur- und Fremdsprachenunterricht vgl. z.B. DonAth (2010), Weissenburger (2006, 2007), im muttersprachlichen Polnischunterricht z.B. PEZDA (2011:93-96). Ein Beispiel für den Einsatz des Blogs im Literaturunterricht ist Tanjozas Weblog http://tanjoza.wordpress.com/2008/ 11/10/blog-im-literaturunterricht/ (20.11.2011); zum Erfahrungsaustausch der Lehrer über Blogs im Unterricht vgl. z. B. http://lernwolke.de/2010/03/26/ blogsim-unterricht-erste-erfahrungen/ (20.11.2011). Einfache und allgemeine Tipps zum Einsatz von Blogs im Unterricht befinden sich auch auf http://www.elba. ethz.ch/services/blogs (20.11.2011).

20 http://literablogtur.blog.onet.pl (20.11.2011). 
Beginn des Wintersemesters 2010/2011 eingerichtet. Ein neues Thema wird ungefähr ein Mal im Monat von der Lehrperson angegeben bzw. von den Lernenden vorgeschlagen. Die Beiträge beziehen sich vor allem auf die im Literaturunterricht behandelten Inhalte: auf literaturwissenschaftliche Probleme, Epochen, Pflichtlektüren, aber auch auf andere literaturbezogene Themen. Das Blog begann mit dem Thema ,Gender und Queer Studies'. Nachdem der wissenschaftliche Hintergrund und die Forschungsfelder der ,Gender und Queer Studies“ im Unterricht besprochen worden waren, sollten die Student(inn)en diese Problematik in ihnen bekannten literarischen Werken und Filmen aufspüren. Im Laufe des Wintersemesters wurden noch zwei weitere Themen behandelt: die avantgardistische Lyrik und Frauenbilder in Literatur und Film. Im Sommersemester äußerten sich die Bloggerinnen und Blogger über literarische Männerfiguren, Die Blechtrommel, und die Widerspiegelung der ,großen " politischen Geschichte in literarischen Familiengeschichten. Die Student(inn)en setzten sich mit sehr interessanten Texten und Filmen auseinander und nahmen sowohl kritisch als auch anerkennend, aber vor allem sehr persönlich und emotionell Stellung. Sie suchten selbstständig nach zusätzlicher Sekundärliteratur und zitierten wissenschaftliche Definitionen, zogen originelle Vergleiche zwischen literarischen Figuren, offenbarten ästhetische Sensibilität, übten sich in Kritikfähigkeit, zitierten Literaturkritiken, präsentierten und verteidigten überzeugend eigene Meinungen, lobten, widersprachen, scherzten und kritisierten in ihren Kommentaren. Die Lehrperson kommentiert die Texte, ergänzt sie um Hinweise auf weiterführende Literatur. Die interessantesten Blog-Äußerungen werden im Unterricht besprochen, was Gelegenheit zu weiteren Diskussionen und Literaturhinweisen gibt. Das Blog belebt, bereichert und ergänzt die Arbeit im Literaturunterricht. Das Bloggen entwickelt neben der literarischen Rezeptionskompetenz auch die Produktions- und Handlungskompetenz. Es bietet auch Gelegenheit, auf literarische Texte emotionell zu reagieren. In der indirekten Online-Kommunikation äußern sich die Bloggerinnen und Blogger viel ungezwungener als im Unterricht.

Das Bloggen hat eine sehr wichtige motivierende Funktion, indem es der neuen Lernergeneration zeigt, dass die Literatur viele Leser nach wie vor persönlich betrifft, Emotionen und Intellekt gleichermaßen anspricht und zum Nachdenken anregt. Neben den literarischen Teilkompetenzen entwickelt es auch die Sprachkompetenz (das kreative Schreiben) und die Medienkompetenz. Das LiteraBLOGtur hat noch mehr Lebendigkeit und Spontaneität in den Literaturunterricht gebracht und die Student(inn)en überzeugt, dass Literatur kein toter Lerngegenstand ist, sondern zum Alltag ge- 
hört und als Mittel der ,Daseinserhellung ' ein wichtiger Faktor der Persönlichkeitsbildung ist. Dieses affektive Lernziel ist heute außerordentlich wichtig. In einer Zeit, in der das enzyklopädische Wissen per Mausklick immer und überall zugänglich ist, ist die Herausbildung wünschenswerter Haltungen wie des Bedürfnisses nach Wissen, nach ständiger Weiterbildung, nach ästhetischem Genuss und nach moralischer Selbstreflexion nicht weniger wertvoll als die Kenntnis von Daten, Fakten und Interpretationen.

$\mathrm{Zu}$ den Nachteilen der Blogs gehört der große Arbeits- und Zeitaufwand seitens der Lehrkraft bei der Gestaltung und Betreuung des Blogs. Notwendig ist auch das technische Know-how für das Gestalten des Blogs; eine Voraussetzung, die nicht immer erfüllt werden kann. Die im 5. und 6. Abschnitt dargestellten handlungsorientierten Methoden sind aus didaktischer und lernpsychologischer Sicht hocheffektiv, denn sie ermöglichen ein dauerhaftes Behalten des Lernstoffes dank Elaboration, sie geben Gelegenheit zur emotionellen Anteilnahme und zum ästhetischen Erleben des literarischen Werkes. Aus literaturwissenschaftlicher Sicht wird ihnen die mangelnde ,Wissenschaftlichkeit‘ vorgeworfen: die Vernachlässigung der avancierten theoretischen Erwägungen und interpretatorischen Nuancen zugunsten der eigenen Produktivität und Kreativität der Lerner. Sie sind auch zeitaufwendig bei der Durchführung im Unterricht: Die Arbeit an einer Literaturepoche mit Einsatz von Übungen dauert statt einer einzelnen Vortragsstunde eine doppelte Seminarstunde, und für die handlungsorientierte Arbeit an einem literarischen Werk braucht man mehr Zeit. Ein kurzer Literaturlehrgang ist dafür nicht ausreichend. Die Didaktisierung des Lernstoffes und die Vorbereitung der Unterrichtsmaterialien sind auch arbeitsaufwendig. Diese Nachteile führen teils dazu, den Einsatz dieser Methoden nicht vornehmen zu können oder nicht vornehmen zu wollen.

\section{Andere Verfahrensweisen}

Der einfachste Weg, sich an die heutige didaktische Situation anzupassen, ist, die Kursinhalte $\mathrm{zu}$ reduzieren und die Lehrveranstaltungen in polnischer Sprache abzuhalten. Der Verzicht auf viele Aspekte der deutschen Literaturgeschichte ist vor allem im berufsorientierten Bachelor-Studium sichtbar. Die Literatur von ihren Anfängen (althochdeutsche Literatur) bis zum Barock wird an Berufshochschulen (PWSZ) und Fremdsprachenkollegs (NKJO), aber auch an anderen Hochschultypen (wie z. B. Technischen Hochschulen) nicht behandelt. Auch in Bezug auf die behandelten Epochen (Aufklärung - Gegenwartsliteratur) ist die Kürzung der Lerninhalte zu 


\section{Ewa Turkowska}

bemerken. Mittlerweile ist es allgemein üblich, umfangreiche Ganzschriften als Pflichtlektüren wegzulassen. Wegen der anspruchsvollen Sprache, der Länge und der Komplexität der Problematik werden solche Kanonwerke wie z. B. Lessings Miß Sara Sampson, Minna von Barnhelm, Goethes Götz von Berlichingen, Schillers Don Carlos oder Maria Stuart, Fontanes Effi Briest, Hauptmanns Die Weber, Manns Zauberberg, Doktor Faustus oder Grass' Die Blechtrommel nicht mehr ausführlich analysiert und interpretiert, sondern in Vorlesungen kurz besprochen oder in Seminaren in Ausschnitten gelesen. Dieser Trend ist auch in anderen Ländern zu verzeichnen. Für den heutigen fremdsprachlichen Literaturunterricht in Polen trifft dasselbe $\mathrm{zu}$, was E. Paefgen vor dreizehn Jahren über den muttersprachlichen Literaturunterricht in Deutschland bemerkte:

Mit Blick auf die Geschichte des Literaturunterrichts und der -didaktik muss festgestellt werden, dass die Gegenstände des Faches immer einfacher, kürzer und leichter geworden sind. [...] [L]agen die vollständigen und ungekürzten Werke unterschiedlichster Zeiten bis in die 1960er Jahre hinein dem Unterricht in den höheren Klassen zugrunde, so muss für die letzten zwanzig bis dreißig Jahre eine kontinuierliche Erleichterung des literarischen Lernprozesses konstatiert werden. [...] Nicht immer werden vollständige Dramen- oder Romantexte gelesen, sondern Auszüge aus mehreren Werken [...]. Wenn ganze Texte gelesen werden, werden nicht selten einfachere oder kürzere Texte gewählt: statt Th. Manns Buddenbrooks eher Tonio Kröger oder Der Tod in Venedig; statt Franz Kafkas Der Prozess oder Das Schloss eher Das Urteil oder Die Verwandlung; statt Uwe Johnsons Mutmaßungen über Jakob eher Heinrich Bölls Die verlorene Ehre der Katharina Blum; statt Robert Musils Verwirrungen des Zöglings Törle $\beta$ lieber Max Frischs Homo Faber, statt Gottfried Benns Gehirne eher Kurzgeschichten von Wolfgang Borchert. Sowohl für die Grundschule als auch für Sekundarstufe I und II werden die Leseanforderungen - was Komplexität und Länge angeht - gesenkt. [...] [Es] wird tendenziell weniger, [es] werden kürzere und [es] werden verständlichere Texte gelesen. (PAEFGEN 1999:152f.).

Vorlesungen und Seminare werden an vielen Instituten entweder zweisprachig oder nur auf Polnisch abgehalten. Das erlaubt, einen umfangreichen Lernstoff zeitökonomisch und ohne großen Arbeitsaufwand zu vermitteln. Das zu bewältigende Pensum im Literaturkurs erzwingt eine solche Lösung. Das bedeutet aber zugleich, die Sprachbildung der Studierenden nicht zu berücksichtigen. Die Entscheidung, welches Ziel wichtiger ist - das Literaturwissen oder die Entwicklung der Sprachkompetenz -, ist sehr schwierig. Das betrifft insbesondere diejenigen germanistischen Institute, die sich die Ausbildung des literaturwissenschaftlichen Nachwuchses zum Ziel setzen und die Lerner vom Studienbeginn an in die Methoden der wissenschaftlichen Arbeit einführen möchten. 
Diskussionen auf internationalen Tagungen und Fachbeiträge aus Deutschland, Ungarn, der Tschechischen Republik und Slowenien zeugen davon, dass im Ausland ähnliche Wege beschritten werden, um die literarische Bildung an die heutige Situation anzupassen. Eine größere Bedeutung misst man der didaktisch-methodischen Gestaltung des Unterrichtsprozesses bei. Die Handlungs- und Produktionsorientierung im Literaturunterricht beginnt die Hochschuldidaktik zu dominieren, große Aufmerksamkeit schenkt man der Intermedialität, darunter der Filmdidaktik und der Unterstützung der literarischen Lernprozesse mit Hilfe neuer Medien (vgl. z.B. BIAŁEK / KAROLAK 2007; BIAŁEK / HuSZCZA 2009; KOCHANOWSKA-NIEBORAK / PŁOMIŃSKAKRAWIEC 2012:275-398).

Das heutige Germanistikstudium ist heterogen geworden, diverse Hochschultypen verfolgen unterschiedliche Bildungsziele. In dieser didaktischen Situation existiert kein einheitliches Modell der literarischen Bildung und kein Pauschalrezept für den Literaturunterricht mehr, das alle Hochschulen mit gleichem Erfolg anwenden könnten. Jedes didaktische Verfahren muss im Hinblick auf die Bildungsziele der jeweiligen Institute erwogen, den lokalen Bedingungen sowie der Lehrtradition angepasst werden. Gleichzeitig ist auch das methodische Repertoire mit dem neuen Medienangebot komplexer geworden, man kann daher in der gegenwärtigen Situation mehr als zuvor einen motivierenden, kreativen Literaturunterricht gestalten.

\section{Literatur}

Abraham, Ulf / KePser, Matthis (2006): Literaturdidaktik Deutsch. Eine Einführung. Berlin.

Allroggen, AnTJe (2006): ,German Studies ‘ in der Krise: http://www.dradio.de/dlf/ sendungen/campus/482120/ (20.11.2011).

BAACKE, DiETER (1997): Medienpädagogik. Tübingen.

BAuER, Christian (1996): Das Land der Griechen mit der Seele suchend... Die Weimarer Klassik. Videofilm. Bonn.

BiateK, Edward / Huszcza, Krzysztof (eds.) (2009): Förderung der Lesekompetenz im schulischen und universitären Bereich. Dresden/Wrocław.

BiAŁEK, EdWARD / KAROLAK, CZESŁAW (eds.) (2007): „Schuhnummer oder Leben!“ Beiträge zur Literaturdidaktik und zum kinder- und jugendliterarischen Schriftum. Dresden/Wrocław.

Bickenbach, Matthias / MAYe, Harun (2009): Metapher Internet. Literarische Bildung und Surfen. Berlin.

BOESKEN, GESINE (2010): Literarisches Handeln im Internet: Schreib- und Leseräume auf Literaturplattformen. Konstanz. 
Ewa Turkowska

Burwitz-Melzer, Eva (2003): Allmähliche Annäherungen. Fiktionale Texte im interkulturellen Fremdsprachenunterricht der Sekundarstufe I. Tübingen.

- (2007): Ein Lesekompetenzmodell für den fremdsprachlichen Literaturunterricht. In: BREDELla, LothaR / HALLET, WolfGANG (eds.): Literaturunterricht, Kompetenzen und Bildung. Trier (=Handbücher zur Literatur- und Kulturdidaktik 2), 127-158.

DAWIDOWSKI, CHRISTIAN (2009): Literarische Bildung in der heutigen Mediengesellschaft. Eine empirische Studie zur kultursoziologischen Leseforschung. Frankfurt (M.).

DONATH, REINHARD (2010): Blogs im Englischunterricht. Neue Möglichkeiten für das Lehren und Lernen. In: Computer+Unterricht 77:12-16.

FeHR, Johannes / Gond, WALTER (eds.) (2002, 2003): Schreiben am Netz. Literatur im digitalen Zeitalter. 2 Bde. Innsbruck.

FRESE, JULIA (2011): Germanistik im Krisengespräch. In: Litlog. Göttinger eMagazin für Literatur - Kultur - Wissenschaft:http://www.litlog.de/germanistik-im-krisengesprach/ (20.11.2011).

HAAS, GERHARD (1997): Handlungs- und produktionsorientierter Literaturunterricht. Theorie und Praxis eines anderen Literaturunterrichts für die Primar- und Sekundarstufe. Seelze.

HÄRLE, GERHARD / RANK, BERNHARD (eds.) (2004): Wege zum Lesen und zur Literatur. Baltmannsweiler.

- (eds.) (2008): „Sich bilden, ist nichts anders, als frei werden. “Sprachliche und literarische Bildung als Herausforderung für den Deutschunterricht. Baltmannsweiler.

HurRelmann, Bettina / BeCKer, SusAnNe (eds.) (2003): Kindermedien nutzen. Medienkompetenz als Herausforderung für Erziehung und Unterricht. Weinheim und München.

JAZBEC, SAŠA (2009): Man taucht in eine andere Welt ein... Lesestrategien beim Lesen fremdsprachlicher Literatur. Frankfurt (M.).

Jost, Eva (2010): Zwischen Schiller und Netzkultur. In: magazin-deutschland.de: http://www.magazin-deutschland.de/de/artikel/artikelansicht/article/zwischen-schillerund-netzkultur.html (20.11.2011)

Kochanowska-NieboraK, AnNa / PŁomińsKa-Krawiec, Ewa (eds.) (2012): Literatur und Literaturwissenschaft im Zeichen der Globalisierung. Frankfurt (M.)/Berlin/ Bern u. a.

KuMSCHLIES, KiRSTEN (2008): Es war sehr schön, in dir zu leben... Literarische Kompetenz und szenische Interpretation. Frankfurt (M.).

MAHNE, Nicole (2007): Transmediale Erzähltheorie. Eine Einführung. Göttingen.

Moser, HeINZ (2010): Einführung in die Medienpädagogik: Aufwachsen im Medienzeitalter. Wiesbaden.

PAEFGEN, ElisABETH K. (1999): Einführung in die Literaturdidaktik. Stuttgart.

Pezda, Aleksandra (2011): Koniec epoki kredy. [Das Ende der Kreide-Ära]. Warszawa. 
PiestraK-Demirezen, Dorota (2009): Hypermediale Fiktionen: zu einem Phänomen der digitalen Literatur. Frankfurt (M.).

Segeberg, Harro / Winko, Simone (eds.) (2005): Digitalität und Literalität: zur Zukunft der Literatur. München.

SIMANOWSKI, ROBERTO (2002): Interfictions. Frankfurt (M.).

SPINNER, KASPAR H. (2006): Literarisches Lernen. In: Praxis Deutsch 33:6-16.

STASIAK, Halina (1995) (ed.): Curriculum für Fremdsprachenkollegs. Warszawa.

Süss, Daniel / Lampert, Claudia / Wijnen, Christine W. (2010): Medienpädagogik. Wiesbaden.

Tränen des Vaterlandes oder das literarische Barock (1999). Video-Film. Bonn.

TURKowsKa, EwA (2000): Literatur in der Deutschlehrerausbildung. In: Kollegzeitung 1:9-12.

- (2006): Literaturvermittlung in der Deutschlehrerausbildung. Praxis und Theorie. Radom.

- (2011): Einführung in die Literaturwissenschaft. Ein Handbuch für Germanistikstudenten. Dresden/Wrocław.

Vogt, Jochen (2008): Einladung zur Literaturwissenschaft. Paderborn.

WEISSENBURGER, CRISTIAN: (2006): Blogs für die Leseförderung. Eine Chance für den Literaturunterricht. In: merz. zeitschrift für medienpädagogik 1:65-67.

- (2007): Weblogs im Deutschunterricht. In: Lehren und Lernen 11:8-10.

WIENER, DAWID (2009): Jak mózg się przegrzewa. [Wie das Gehirn überheizt wird]. In: Gazeta Wyborcza, 14.-16.8.2009:30f. 